\title{
Implementation of Fixed and Floating Point Square Root Using Nonrestoring Algorithm on FPGA
}

\author{
Anuja Nanhe, Gaurav Gawali, Shashank Ahire, and K. Sivasankaran
}

\begin{abstract}
Square root is one of the fundamental arithmetic operations used in recent generation processors. In this paper, we present pipelined architecture to implement 8 bit fixed and floating point square root in Field Programmable Gate Array (FPGA) using modified nonrestoring square root algorithm. This algorithm has been optimized by eliminating a number of elements without compromising the precision of the square root and the remainder. It is an improvement over nonrestoring algorithm as it uses only subtract operation and append 01 instead of add operation and append 11. Here the basic building block is Controlled Subtract Multiplex (CSM). By using this module, the algorithm can be designed for any number of input bits. This strategy offers an efficient use of hardware resources. The modified non restoring algorithm is designed using Verilog HDL and implemented on ALTERA cyclone II FPGA. The implementation results show reduced area in terms of logic elements when compared to restoring algorithm.
\end{abstract}

Index Terms-Controlled subtract multiplex, fixed point, FPGA, nonrestoring, square root, verilog HDL.

\section{INTRODUCTION}

The square root function is widely used in computer graphics, image and signal processing, statistics, communications and scientific calculation applications. Due to complications involved in implementation of square root algorithms, its design in digital system has always been a bottleneck. The basic operations like addition and subtraction are easy to implement in an FPGA because synthesis tools have optimized addition/subtraction units based on FPGA architecture. Multiplication, division and square root are complex operations; square root in particular, is computationally intensive as it involves convergence and approximation techniques [1]. Many algorithms/methods have been developed to implement it on FPGAs. But there is a need of an algorithm which should be more efficient in terms of time, speed and on-chip area.

Existing techniques for evaluating square root function in computer arithmetic, such as direct methods, algorithms based on Newton-Raphson formula, radix 2 Sweeney Robertson Tocher (SRT) redundant and nonredundant algorithms. Also, there are methods based on normalization techniques and approximations by real functions [2]. Newton Raphson algorithm needs multiplication and addition or subtraction at each iteration. This is performed using Wallace tree adder followed by carry propagate adder. This procedure requires large number of gate counts which is not feasible to implement on FPGA. SRT redundant and nonredundant

Manuscript received February 9, 2013; revised May 31, 2013.

The authors are with School of Electronics Engineering, VIT University, Vellore (e-mail:\{anujananhe26, gawali.gaurav, shashank.ahire9, ksivasankaran.vlsi\}@gmail.com). algorithms result in wrong value at last digit position [3]. To overcome these difficulties, digit by digit algorithm has been designed which is classified as restoring and nonrestoring algorithm. Both algorithms are compared with Look Up Table (LUT) implementation using different bit widths [4] Nonrestoring algorithm has minimum hardware resources as it does not store remainder and so it is easy to implement on FPGA as compared to restoring algorithm. Yamin and Wanming [5], [6] have introduced nonrestoring algorithm with fully pipelined and iterative version that requires neither multipliers nor multiplexers. They have introduced the carry save adder (CSA) and carry propagate adder (CPA) which improves speed at the cost of more hardware resources. A classical design on Controlled Add-Sub (CAS) module with moderate hardware resources reduces area and delay [7]. A flexible approach which makes use of memory, shift elements and multipliers is introduced in which as the input number increases; number of cycles required for the calculation of square root also increases [8]. Sajid, Ahmed and Ziavras [9] have proposed nonrestoring square root algorithm using add/subtract-shift. The throughput of this system has been improved by pipelining. Tole Sutikno [10] has proposed modified square root algorithm for fixed point number using VHDL whereas Buradkar and Zode [11] have extended this work to signed number using VHDL.

In this paper, we study restoring and modified nonrestoring square root algorithm and implement them using pipelined architecture in Verilog HDL [12]. This approach uses CSM as a basic building block. The block has been modified for implementing restoring algorithm. These blocks have been optimized to reduce the number of logic cells utilized. The performance is compared based on logic elements and power consumption using ALTERA Cyclone II FPGA.

\section{RESTORING AND MODIFIED NONRESTORING SQUARE ROOT ALGORITHM}

\section{A. Restoring Algorithm}

The steps for calculating square root using restoring algorithm are as follows [10]:

Step 1: If $\mathrm{M}$ is a $2 \mathrm{n}$ bit number then divide it in a group of 2bits.

Step 2: Subtract 1 from the first 2 bits (starting from MSB)

Step 3: If the result of the subtraction is positive then the developed root (quotient) is ' 1 ' otherwise ' 0 '.

Step4 : If the result is negative, write it as it is. Restore the wrong guess by appending ' 01 ' and quotient.

Step 5: Take the next two bits.

Step 6: Append '01' (to be subtracted from next two bits of dividend) and quotient to subtract from the remainder. 
Step7: If the result of subtraction is negative write previous remainder as it is, leaving the first 2 bits.

Step 8: Every time quotient has to be updated while appending ' 01 '.

Step 9: Continue the steps until the group of two bits end.

The following Fig. 1 shows the example of restoring algorithm to calculate square root:

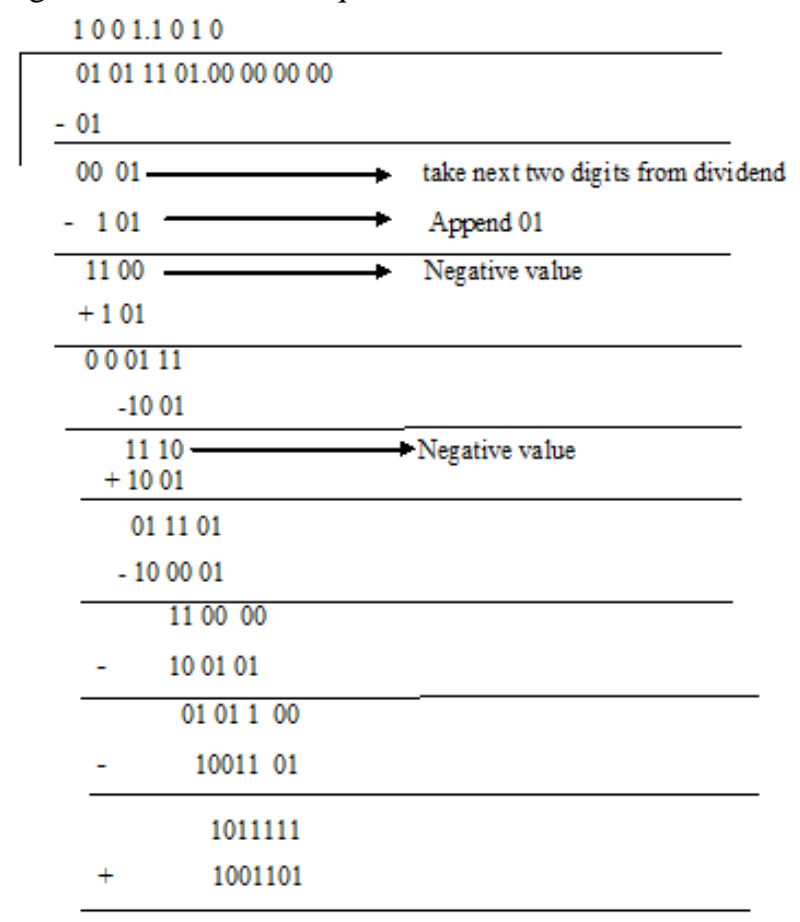

\begin{tabular}{cc}
01011000 \\
$-\quad 010011001$ \\
\hline & 00001011100 \\
$-\quad 100110101$ \\
\hline
\end{tabular}

Fig. 1. The example of restoring algorithm to solve square root.

\section{B. Modified Nonrestoring Algorithm}

A small modification in nonrestoring algorithm makes calculation faster. It uses only subtract operation and appends " 01 ". It uses $n$ stage pipelining to find square root of $2 \mathrm{n}$ bit number. The following algorithm describes the modified nonrestoring square root algorithm.

Step 1: Start

Step 2: Initialize the radicand (M) which is $2 \mathrm{n}$ bit number. Divide the radicand in two bits beginning at decimal point in both directions.

Step 3: Beginning on the left (most significant), select the first group of one or two bits. (If $n$ is odd then first group has one bit, else two bits.)

Step 4: Select the first group of bits and subtract' 01' from it. If borrow is zero, result is positive then quotient is 1 otherwise it is 0 .

Step 5: Append 01(to be subtracted next two bits of dividend) and quotient to subtract from remainder of previous stage.

Step 6: If result of subtraction is negative, write previous remainder as it is and quotient is considered as 0 , else write the difference as remainder and quotient as 1 .

Step 7: Repeat step 5 and step 6 until end group of two bits. Step 8: End.
The following Fig. 2 shows the example of nonrestoring algorithm to calculate square root:

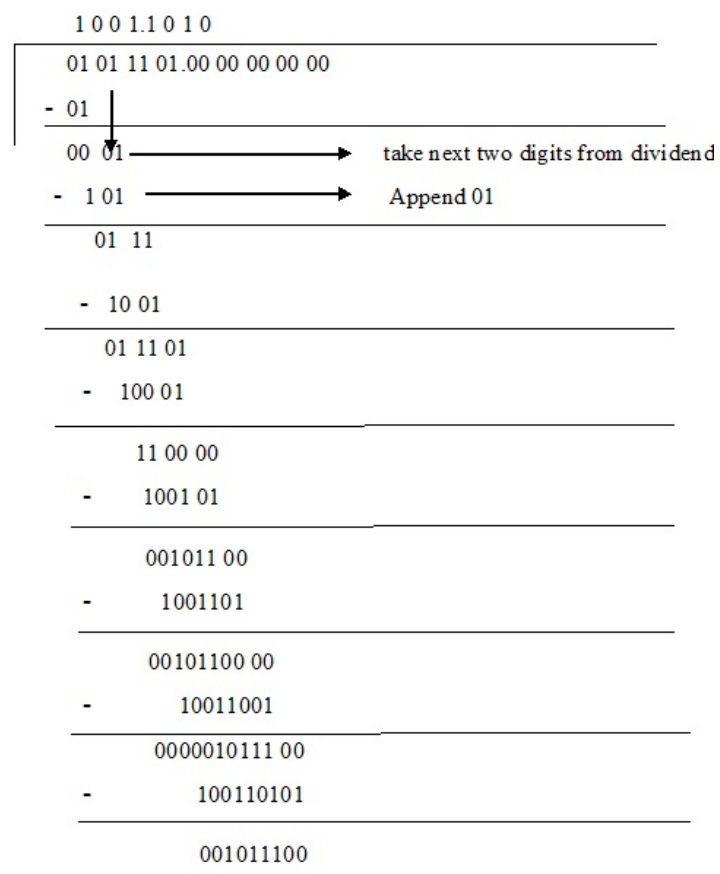

Fig. 2. The example of modified nonrestoring algorithm to solve square root.

\section{BASIC BUILDING BLOCK FOR MODIFIED NONRESTORING} ALGORITHM

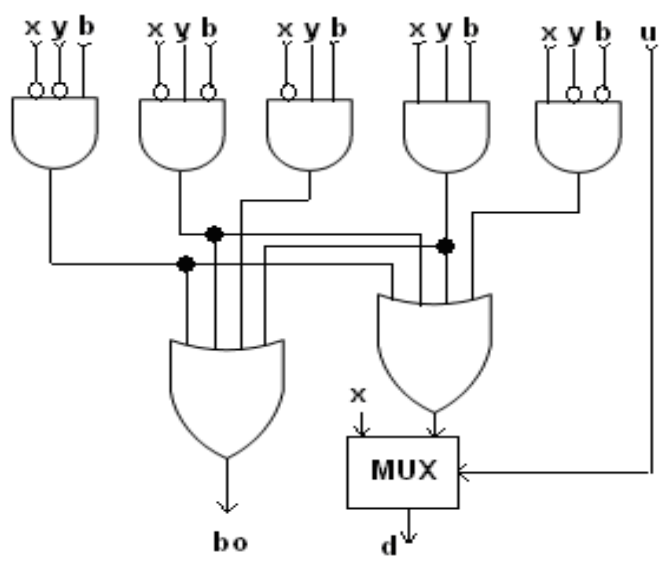

Fig. 3. Internal structure of CSM block[10].

From Fig. 3, it is clear that Controlled Subtract Multiplex(CSM) is a combination of full subtractor and 2:1 multiplexer. Multiplexer is used to select one of the inputs based on one bit quotient which acts as a select line for multiplexer.

From the algorithm, it can be concluded that if the result of subtraction is negative (which will set the borrow bit to 1 ), quotient " $u$ " is selected as 0 which ultimately selects the input " $x$ " for the next iteration. Also if the result of subtraction is positive which gives 0 as a borrow, quotient is selected as 1 . This sets select line to 1 which gives difference " $d$ " as input for next iteration.

Inputs to the CSM block are $x, y, b$ and $\mathrm{u}$ whereas $d$ and $b 0$ (borrow) are outputs.

If $b O=0$, then $d<=x-y$ - $b$ else $d<=x$. 
As CSM includes full subtractor having $x, y$ and $b$ as its inputs, it works as follows:

1) It performs subtraction : $x-y-b$

2) If the result of subtraction is positive, we get $b 0=0, u$ $=1$

and $d=x-y-b$

3) If the result of subtraction is negative, we get $b 0=1$,

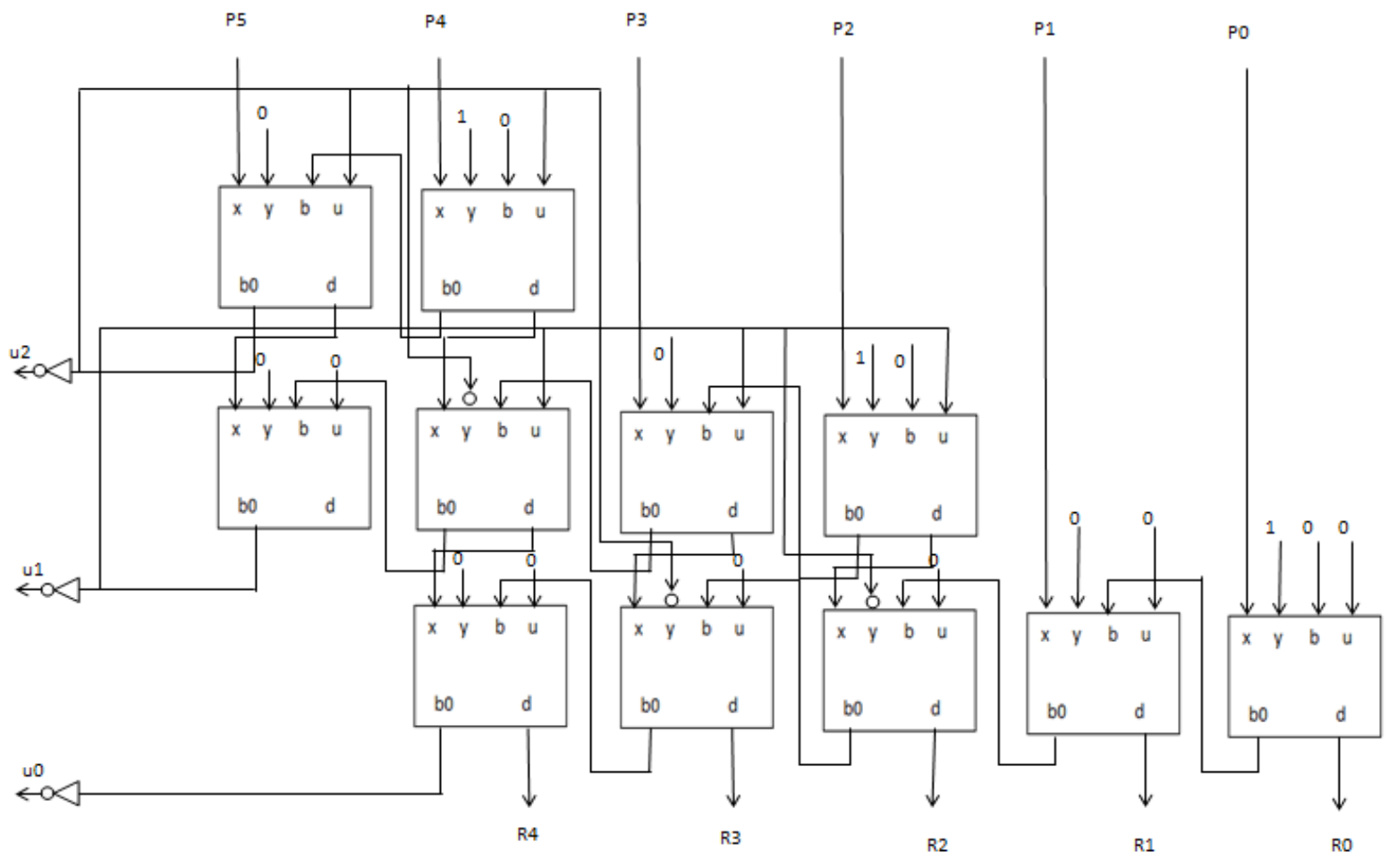

Fig. 4. Pipelined structure of 6 bit unsigned square root number.

The generalization of simple implementation of modified nonrestoring digit by digit algorithm for unsigned 6 bit square root is shown in Fig. 4. For 6 bit input number, 3 bit quotient $\left(\mathrm{u}_{2} \mathrm{u}_{1} \mathrm{u}_{0}\right)$ is obtained as answer.

Each row of the circuit executes one iteration of nonrestoring digit by digit square root algorithm, where it only uses subtract operation and appends ' 01 '. In the pipelined structure using CSM block, some input patterns are fixed. So , the design can be optimized by minimizing the boolean equations of b0 and d. It can be implemented by modifying CSM block.The specialized entities A,B,C,D,E,F,G, H and I are derived from it and are defined as follows:

i. For csmA, $y b u=100$

iv. For csmD, $y b=10$

$$
\begin{aligned}
& b 0=\bar{x} \\
& d=\bar{x} \cdot \bar{u}+x . u
\end{aligned}
$$

v. For csmE, $y=0$

$$
\begin{aligned}
& b 0=\bar{x} \cdot b \\
& d=\bar{x} \cdot b \cdot \bar{u}+\bar{B} x+x \cdot u
\end{aligned}
$$

$u=0$ and $d=x$

Hence, the following boolean equations are obtained for borrow $(b 0)$ and difference $(d)$.

$$
\begin{gathered}
b 0=(\bar{x} \cdot y)+(b \cdot \bar{x})+(b y) \\
d=(\bar{x} \cdot y \cdot \bar{b} \cdot \bar{u})+(\bar{x} \cdot \bar{y} \cdot b \cdot \bar{u})+(x \cdot \bar{y} \cdot \bar{b})+(x \cdot u)+(x \cdot y \cdot b)
\end{gathered}
$$




$$
b 0=b
$$

ix. For csmI, $x y b u=0100$

$$
\begin{aligned}
& b 0=1 \\
& d=1
\end{aligned}
$$

The optimized hardware implementation of modified non restoring 8 bit square root is generalized in Fig. 5

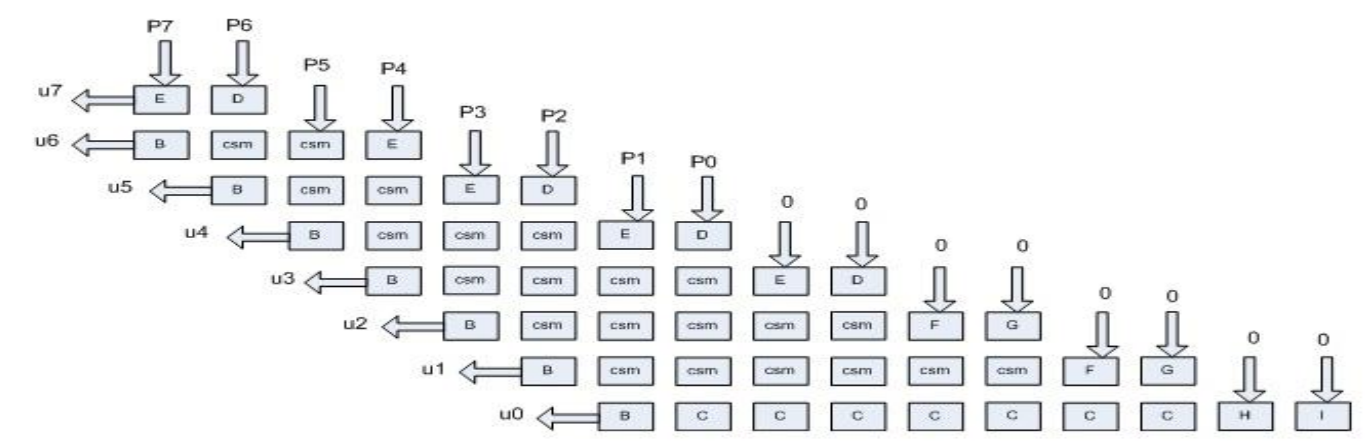

Fig. 5. Pipelined structure of optimized 8 bit unsigned square root number.

TABLE I: LOGIC ELEMENTS USAGE

\section{RESULTS AND DISCUSSIONS}

The simulation results of 8 bit fixed and floating point

\begin{tabular}{|c|c|c|}
\hline \multicolumn{3}{|c|}{ Messages } \\
\hline $\mathbf{+}^{-1}$ / $/$ sqrt_8_tst/p & 11100001 & $11111111,00001001,00010000,10000000,11100001$ \\
\hline $\mathbf{t}^{-} /$/ $/$sqrt_8_tst/r & 000000 & 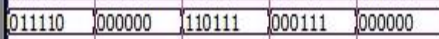 \\
\hline $\mathbf{t}^{-} /$/sqrt_ 8 _tst/u & 1111 & 1011 \\
\hline
\end{tabular}
square root using modified nonrestoring algorithm is shown in Fig. 6 and Fig. 7 respectively.

Fig. 6. Simulation result of 8 bit fixed point square root using modified nonrestoring algorithm.

The notations used in waveforms are " $p$ " for dividend," $u$ " for quotient and " $r$ " for remainder. In case of floating point quotient, first four bits starting from MSB represent number before decimal point and the remaining bits represent number after decimal point.

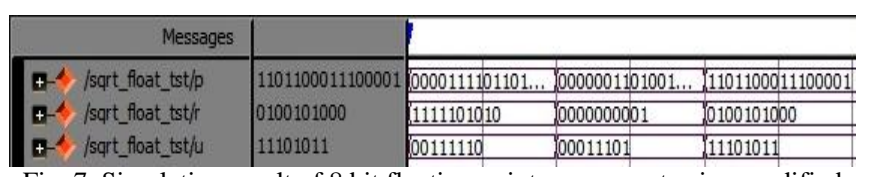

Fig. 7. Simulation result of 8 bit floating point square root using modified nonrestoring algorithm

The CSM block is modified and implemented for restoring algorithm by adding full adder which serves the purpose for restoring algorithm. This addition of adder block leads to larger area as the logic elements increase. The comparison of logic elements utilized for implementation of 8 bit restoring and modified nonrestoring square root algorithm is shown in Table I.

The modified nonrestoring algorithm has been optimized by simplifying CSM block which reduces the number of hardware resources. This comparison of logic element usage of modified nonrestoring algorithm with and without optimization is shown in Table II.

The implementation of 8 bit fixed point modified nonrestoring algorithm using Altera Cyclone II FPGA requires 50 logic elements (LE) with optimization and 71 LEs

\begin{tabular}{|c|c|c|c|}
\hline \multirow{2}{*}{\multicolumn{2}{|c|}{ Types of Algorithms }} & \multicolumn{2}{|c|}{ LE Usage } \\
\hline & & $\begin{array}{l}\text { Without seven } \\
\text { segment }\end{array}$ & $\begin{array}{c}\text { With seven } \\
\text { segment }\end{array}$ \\
\hline \multicolumn{2}{|r|}{ Restoring Algorithm } & 61 & 74 \\
\hline \multicolumn{2}{|c|}{ Modified nonrestoring Algorithm } & 50 & 64 \\
\hline \multicolumn{4}{|c|}{$\begin{array}{l}\text { The Table II shows comparison of logic elen } \\
\text { for implementation of } 8 \text { bit nonrestoring } \\
\text { algorithm with and without optimization. }\end{array}$} \\
\hline \multicolumn{4}{|c|}{$\begin{array}{c}\text { TABLE II: LOGIC ELEMENTS USAGE } \\
\end{array}$} \\
\hline \multirow{2}{*}{\multicolumn{2}{|c|}{ Types of Algorithms }} & \multicolumn{2}{|c|}{ LE Usage } \\
\hline & & nization & pptimization \\
\hline & $\begin{array}{l}\text { ified nonrestoring } \\
\text { Algorithm } \\
\end{array}$ & & 71 \\
\hline \multicolumn{4}{|c|}{$\begin{array}{l}\text { The performance of this square root system can also be } \\
\text { measured by different types of powers consumed by the } \\
\text { system. Table III shows the status of Power Play Power } \\
\text { Analyzer. }\end{array}$} \\
\hline \multicolumn{4}{|c|}{ TABLE III: POWER PLAY POWER ANALYZER REPORT } \\
\hline No & $\begin{array}{l}\text { PowerPlay Power } \\
\text { Analyzer Report }\end{array}$ & $\begin{array}{l}\text { with optimization } \\
\qquad(\mathrm{mW})\end{array}$ & $\begin{array}{c}\text { bit } \\
\text { without } \\
\text { optimizati } \\
\text { on }(\mathbf{m W})\end{array}$ \\
\hline 1 & $\begin{array}{c}\text { Total Thermal } \\
\text { Power Dissipation }\end{array}$ & 447.96 & 898.63 \\
\hline 2 & $\begin{array}{l}\text { Core Dynamic } \\
\text { Thermal Power } \\
\text { Dissipation }\end{array}$ & 190.47 & 306.22 \\
\hline 3 & $\begin{array}{l}\text { Core Static Thermal } \\
\text { Power Dissipation }\end{array}$ & 48.06 & 48.97 \\
\hline 4 & $\begin{array}{l}\text { I/O Thermal Power } \\
\text { Dissipation }\end{array}$ & 209.44 & 543.43 \\
\hline
\end{tabular}
without optimization. The number of LEs denotes the actual required hardware resources for circuit implementation. 


\section{CONCLUSION}

This paper presents restoring and modified nonrestoring algorithm for square root calculation. The result shows that restoring algorithm requires more LE's compared to modified nonrestoring algorithm as it stores remainder at each iteration. Thus, optimized nonrestoring algorithm reduces on chip area and pipelining increases the speed performance. The result is extended for square root implementation of 8 bit floating point number and also it can be expanded to larger numbers to solve complicated square root problem in FPGA implementation.

\section{REFERENCES}

[1] Y. Li and W. Chu, "Implementation of single precision floating point square root on FPGAs," FCCM'97, in Proc. IEEE Symposium on FPGAs for Custum Computing Machines, April 16-18, 1997, Napa, California, USA, pp. 226-232.

[2] P. Montuschi and M. Mezzalama, "Survey of square rooting algorithms," IEE Prec. Computers and Digital Techniques, vol. 137, Pt. E, no. I, January 1990, pp. 31- 40.

[3] K. Piromsopa, C. Aporntewan, and P. Chongsatitvatana, "An FPGA implementation of a fixed point square root operation," International Symposium on Communications and Information technologies (ISCIT), 2001.

[4] M. Franke, A. Th. Schwarzbacher, M. Brutscheck, and St. Becker, Implementation of different square root algorithms.

[5] Y. Li and W. Chu, "Parallel-array implementation of a non-restoring square root algorithm," in Proc. IEEE International Conference on Computer Design: VLSI in Computers and Processors, 1997, pp. 690-695.

[6] Y. Li and W. Chu, "A new non-restoring square root algorithm and its VLSI implementation," in Proc. IEEE International Conference, 1996, pp. $538-544$.

[7] S. Samavi, A. R. Rezaee Sadrabadi, and A. Fanian, "Modular array structure for non-restoring square root circuit," Journal of Systems Architecture, vol. 54, no. 10, 2008, pp. 957 - 966.

[8] F. M. del Campo, A. Mprales-Reyes, R. Perez-Andrade, R. Cumplido, A. G. Orozco-Lugo, and C. Feregrino, "A multi-cycle fixed point squre root module for FPGAs," IEICE Electronics Express, vol. 9, no. 11, 2012, pp. 971-977.

[9] I. Sajid, M. M. Ahmed, and S. G. Ziavras, "Pipelined implementation of fixed point square root in FPGA using modified non-restoring algorithm," in Proc. 2010 The $2^{\text {nd }}$ International Conference on

Computar and Automation Engineering, ICCAE 2010, vol. 3, art. no. 5452039, pp. 226-230.

[10] T. Sutikno, "An efficient implementation of the non-restoring square root algorithm in gate level," Internantional Journal of Computer Theory and Engineering, vol. 3, no. 1, February, 2011, 1793-8201.

[11] M. U. Buradkar and P. P. Zode, "A 32-bit signed/unsigned fixed point non-restoring square-root operation using VHDL," International Journal of Computational Engineering Research (IJCER), issn: 2250-3005, National Conference on Architecture, Software system and Green computing.

[12] S. Palnitkar, Verilog $H D L-A$ Guide to Digital and Synthesis, $2^{\text {nd }}$ ed. Pearson, ch. 3 - ch.5, pp. 69-127.
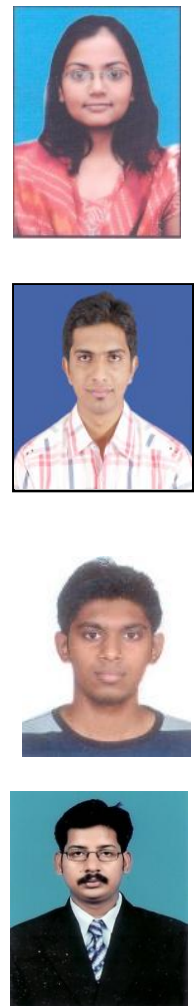

Anuja Nanhe received her B.E degree in Electronics Engineering from Vishwakarma Institute of Technology in Pune University in 2010. From June 2010 to October 2011 she worked as a Member of Technical Staff in Semiconductor Solution Group in KPIT Cummins Infosystem Ltd. Pune. She is currently pursuing MTech (VLSI Design) in School of Electronics Engineering at VIT University, Vellore.

Gaurav Gawali received his Bachelor of Engineering degree in Electronics and Telecommunication Engineering from PICT, University of Pune, India in 2010. In 2011, he worked as a lecturer at K.K. Wagh Engineering College, University of Pune India. Currently, he is pursuing MTech (VLSI Design) in School of Electronics Engineering at VIT University, Vellore.

Shashank Ahire received his Bachelor of Engineering degree in Electronics and Telecommunication Engineering from Maharashtra Institute of Technology, University of Pune, India in 2010. Currently, he is pursuing MTech (VLSI Design) in School of Electronics Engineering at VIT University, Vellore.

K. Sivasankaran is serving as Assistant Professor (Senior) in School of Electronics Engineering at VIT University, Vellore, India. He has 6 years of teaching experience. His research area includes Nanoscale MOS Device Modeling and Simulation and FPGA Based System Design. He has published research article in International Journal and Conferences. He is the member of IEEE, IEEE-EDS, and VLSI Society of India. 